九州大学学術情報リポジトリ

Kyushu University Institutional Repository

\title{
Über Den Dimorphismus Der Epithelzellen Im Mitteldarm Der Seidenraupe (Bombyx Mori L.)
}

Kawaguchi, Eisaku

Sericultural Laboratory, Kyushu Imperial University

https://doi.org/10.5109/22560

出版情報 : 九州大学大学院農学研究院紀要. 3 (3)，pp.47-64，1931-06. Kyushu Imperial University バージョン：

権利関係 : 
Journal of the Department of Agriculture, Kyushu Imperial University, Vol. 3, No. 3
June I, I93r.

ÜBER DEN DIMORPHISMUS DER EPITHELZELLEN IM MITTELDARM DER SEIDENRAUPE (BOMBIX MORI L.)

\author{
Eisaku KaWAGUCHI
}

\title{
EINLEITUNG
}

In Bezug auf den listologischen Bau des Mittelclams der Lepidopterenlarven liegt cinc zahlreiche T.iteratur vor, zu der seit langem von verschierlenen Autoren Bciträgc geliefert worden sind. Nichtdestoweniger gehen die Meinungen über einige Fragen noch inmer auseinander, insbesondere über dic Entstelung der Becherzellen und der Zylinderzellen (auch Calycocyten und Sphaerocyten nach IDEEGLNer genannt).

Die Frage geht, wie man neucrdings aus verschicdenen Angaben schliessen kann, in der Hauptsache darauf hinaus, ob die Becherzellen für eine besondere Art von Drüsenzellen ausser den \%ylinderzellen zu halten sind ${ }^{\top}$ (dimorph), oder ob die Zylinderzeilen ihren Inhalt entlecren und dabei zu Becherzellen sich umwandeln, dic nach kurzer Zeit entweder zugrunde gehen und verschwinden ${ }^{2}$ bzw. sich wieder zu funktionsfähigen Zylinderzelien erneuern ${ }^{3}$ (homomorph).

Die vorliegende Arbeit wurde unternommen, um diese Frage, also ob die Zellen des Mitteldamepithels homonorph oder dimorph sind, nach Möglichkeit aufzuklären.

I Neuerste Angahen: TChang VUxi-TAI (1928 a, 1928 b, $1928 \mathrm{c}, 1929$ a, 1929 b.) HENSON (1929).

$=$ VIRSON $(1897-1893$, IgO5).

3 SimNoda (1926, 1927), BLChMANN (1928). 
lerrn Prof. Dr. Tanasa möchte ich hier nicht nur für die Anregung zu dieser Arbeit, sondern auch für den jederzeit bereitwilligst erteilten Rat meinen herzlichsten Dank aussprechen

\section{Material und Methoden}

Was das Material anbelangt, so bestand es aus sen Seidenraupen einer japanischen Bivoltine-Rasse, "Nilımnischiki." Die Darmkanäle von Larven wurden in allen Wachstumsperioden herauspräpariert und sofort mit Carnors Gemisch und Chamers Flüssigkcit fixiert. Das Gemisch nach Champy gab prägnante Bilder für den feineren Iau der Becherzellen. Zur Embryonenuntersuchung wurden die Eier jeden Tag zweimal auch mit Caknors Gemisch fixiert (24 Stunden lang) und sind unmittelbar nach der Fixierung geschält worden. Zur Konservierung der Materialien im Zustand der Häutung gelangten die Raupen während der ersten Häutung.

Seit ungefähr 34 Stunden vor der Iläutung fressen dic Raupen almählicl immer weniger und 20 Stunden vor dieser bleiben sie ganz ruhig auf ihrem Platz und hören auf zu fressen. Viele Examplare wurden von 34 Stunden an vor der Häutung bis zum Schluss in Abständen von je zwei Stunden konserviert. In Alter von 24-30 Stuncien machen sie die erste Häıtung durch. Zum Hungerexperiment wurden die Raupen in der dritten Wachstumsperiode lange Zeit olne Nahrungszufuh bei $24^{\circ} \mathrm{C}$ gelassen und in Abständen von je 24 Stunden koncerviert. N.ch 24 Stunden I Iungerns slichen sie sehr umruhig nach liutter und spimnen die Seide überall auf ihrem Platz; aber schon 48 Stunden danach haben sie keine Lust mehr, nach Futter zu suchen. Nach I44-stündigem Innger werden sic sehr mager und dann verhungert bald der grössere Teil der Raupen.

Gesclinitten wurde $9 \mu$ dick in Serien längs und quer nach Paraffineinbettung. Gefärbt wurde in der Hauptsache mit Hämatoxylin nach Delafield, Fihrici und Heibenhais und mit Eosin oder Kongorot (Doppclfärbung).

\section{NORMAISTADIUM}

Bevor ich zu meinen eigenen Befunden bei den Embryonen und bei den Raupen während der Häutung und des Hungerzustands übergehe, möchte ich die von verschiedenen Autoren gemachten Beobach- 
tungen über die normalen Raupen angeben. Der Bau des Mitterdarmepithels der normal ernährten Lepidopterenraupen ist von viclen Autoren (Henscilen 1904, Vkrson 1905, Degghele i909, Hastmann 1910 , Newcomer 1915, Foa 1918, Shinoda 1926, i927, Tchang Yung-Tai 1928, 1929, Hexson 1929) genau und eingehend untersucht worden. Ich schliesse mich daher hicr der von ihnen gregebenen Beschreibung an und fügre ausserdem meine eigenen Befunde bei den Seiclenraupen hinzu.

Die histologischen Schnitte lassen erkennen, dass das Epithel des Mitteldarms aus zwei deutlich unterscheidbaren Zelltypen besteht : aus den Becherzellen und aus den mit einem Stäbchensaum versehenen Zylinderzellen (Abb. I, 2).

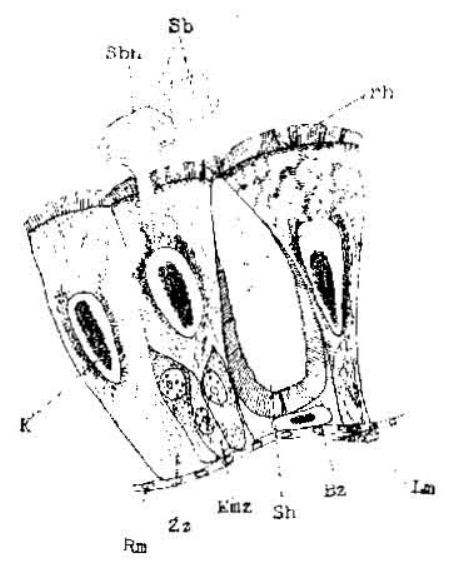

Abb. 1 . Zeilen aus dem virder $n$ Teil des Mitteldarmepithels.

34 Stunden vor der ersten IIäutung.

Bz Becherzelle; Rm Ringmuskel;

Enn Epitheimutterzelle; Sb Sekretblasen;

K. Kern; Sth Sekretblasenlıat;

Lm längsnuskel; Sil Safthohlung;

rh Stäbchensiam; $7 x$ zylinderzelle.

Vergr. 320: 1

Die beiderlei Zellen sind in den ersten Raupenstadien regelmässig gebaut, so dass die Darmwand sehr glatt ist. In dem vorderen Teil des Mitteldarms sind die Epithelzellen im allgemeinen viel kürzer und breiter und färben sich mit Jarbstoffen viel stärker als diejenigen des hinteren Teils. Die Zylinderzellen der früheren Raupenstadien sind fast kubisch und haben cinen verhältnismässig grossen Kern im Zentrum. 


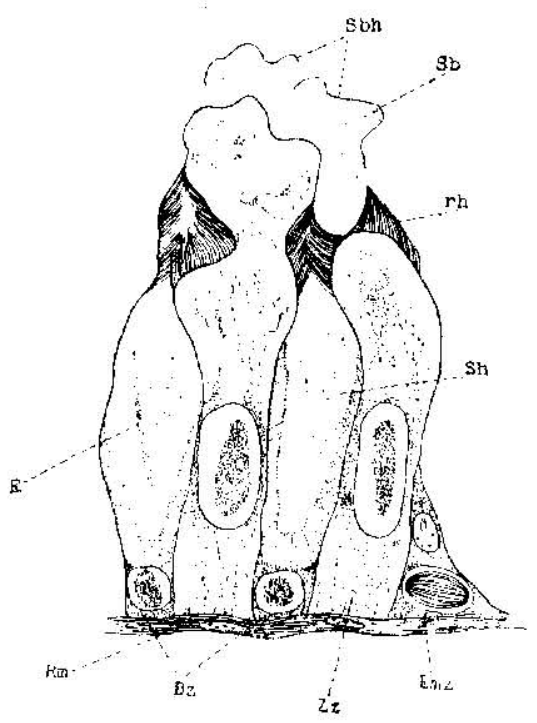

Abb. 2. Zellen aus dem hinteren Teil desselben Epithels f. Finschlüsse;

Jie anderen Bezcichnungen wie Abk. I.

Virgr. $320: 1$

Die Kcrne sind oval, können aber auch ellipsoid oder oblong erscheinen. Sie sind mit Chromatinkörnchen glcichmässig gefüllt. Wir haben einen hellen IIof un das Kernchromatin beobachtet, den DHEGLNer (1909) in seiner Arbeit ïber Dileplita cuphorbiac als Ringhof bezeichnet. Dieser Ringhof kann nur ein durch Carnovsche Flüssigkeit hervorgerufenes Kunstprodukt sein, wie Bucrmax (1928) schon vermutet hat. Dis: Kerne liegen in den fribheren Raupenstadien meistens in der Mitte der Zelle; in den späteren Raupenstadien sieht man sie aber oft an der Zelloberfläche oder ein wenig gegen die Basis verschoben infolge der unregelmässigen und mannigfaltigen Gestalt der betreffenden Zellen. Ihr Plasma, das basophil ist, ist von zahlreichen verschieden starken fadenartigcn Granulationen durchzogen, die die Richtung von der Basis zur Zelloberfäche innehalten.

Solche Granula beobachtet man in besonders reichlicher Menge an den beiden Seiten des Kerns und zwischen der Zelloberfläche und den Kern An der Oberfläche trägt die $Z_{y}$ linderzelle einen deutlichen Stäbchensaum mit einer Basalkörnerreihe und oft mit einer extracytärer an der Basis des Saumș verlaufender Könerreihe. Die Becherzellen erscheinen im allgemeinen in Gestalt von Flaschen, deren Boden an die 
basale Membran stösst (Abb. I und 2). Die Kerne der Becherzellen sind llach-oval im vorderen Teil und rund im hinteren Teil. Sie liegen am Boden der flaschenförmigen Zellen und sind darum von sehr spärlichem basophilem Plasma umgeben. Den anderen, grösseren Tcil der Zallc nimmt eine deutlich ausgebildete Höhlung cin, die den acidophilen Saft cnthält. Darin sind of granuläre oter stäbchenformige Köper eingeschlossen (Abb. 2). An der inneren Seite trägt die Hohlung eine saumformige Schicht, die dem Stäbchensaum ganz ahnlich ist. Diese Schicht ist nach Fixierung mit Champrs Tüssigkeit sehr deutlich erkennbar. Wie die Becherzellen den Inhalt der Hohlung ins Darmlumen entleeren, oder ob sie sich in selber Wcise verhalten wic DeFgener (1909) bei Deilephila euphorbiae beobachtet hat, konnte ich niclit feststellen.

Die Sekreionseischeinungen der Zylincler zellen bei den nomalen Raupen stimmen fast in allen Punkten mit den Bcobachtungen überein, die van Genuchrex ( 1890 ), Dengever (1909) u. a. ausführlich angeben. Die Sekretmasse, die sich unter dem Stäbchensaum anhäuft, buchtet die Zelloberfläche mehr oder weniger stark aus und tritt dann durch den Stabchensaum ins $I$ umen hindurch. Sie nimmt die form einer grossen Blase an, welche sich mit cinem zarten Iläutchen umgibt ( $\triangle \mathrm{bb} .1,2)$. IJäufig kommt es auch vor, das dic einzelnen Sekretblasen nicht zu ejner grossen IBlase zusammenflicssen, sondern es treten mehrere kleine Blasen auf. Bakl geben die Blasen zerplatzend ihren Inhalt an das I)aminnere ab. In anderen Fälen treten die Blasen aus einem zungenformigen Fortsatz der Zelloberfläche heraus. Dicse Art Sekretionsvorgänge gibt es nur in vorderen und im mittleren Teil ries Mitteldarms. Ziemlich anders verhalten sich die Zylinderzellen im hinteren Teil des Mitteldarms. Lei der Abscheidung der Sckretmasse füllen sie sich mit sehr vieJen Vakuolen in eler Zellfront. Diese Vakuolen werden zu grösseren Blasen vereinigt und, indem sie die ganze Zelloberfäche auseinandcrclängen, ins Damlumen ausgestossen (Abb. 2). Dic Sckretcrscheinung ist immer um so vicl lebhafter im hinteren Teil des Mitteldarms als im vorderen, dass die Risse in der Oberfäcle der Seliretionszellen nicht wieder sofort geschlossen werden können. Deswegen ist dic Anordnung des Stäbchensaums schr unregelmässig $(A b b, 2)$. Dabei werclen dic Sekretkugeln ins Lumen auch mit Häutchen umgeben, sie zeigen aber wenig granulären Charakter.

Es ist also anzunehmen, dass die Sekrction im normalen Zustand bei der Seidenraupe eine merocrine ist, wie sie von SHinol) (1926, 
1927) bei einigen Lepidopteren festgestellt worden ist. Ich habe auch bei Bombyx mori beobachtet, dass die Abstossung einzelner Zellen ins Darmlumen, allerdings gelegentlich, stattfinclet (VAN GEHUCHrEN I890, Dregenetr 1908, Bracis 1911).

Was die Frage der Morphogenie der Fpithelzellen anbelangt, bin ich auf keine Übergangsfiguren zwischen Zylinderzellen und Becherzellen oder umgekehrt gestossen, die von VEksON (1897-1898, I905), Shinoda (1926) und Bucmuan (1928) beobachtet worden sind. Häufig werden jugendiliche Becherzellen in den Ejpithehutierzellen (d. h. Nidi) während und kurz nach der Häutung falsch für Übergangsstadien von Becherzellen zu Zylinderzellen gehaten. Die jugendlichon Becherund Zylinderzellen sind noch schmal und haben kleine Kerne und kleine Safthöhlungen in ihrem distalen Teil, worauf ich später eingehen werde.

Dic genannten Autoren haben vielleicht die betreffenden jungen Zellen kur\% nach der Häntung der Raupen bei unkritischer Verwendung des Materials für die gesuchten Übergangsstadien gehalten.

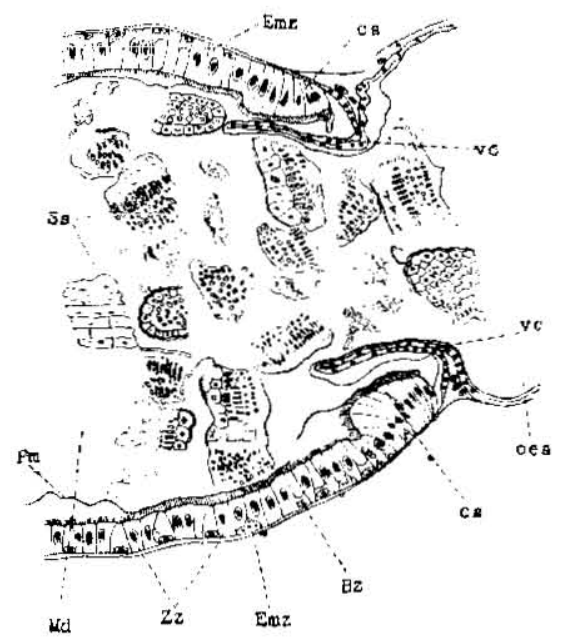

Abt. 3. Längssehnite durch die Cardiaregion. 34 Stunden vor der ersten I läutung.

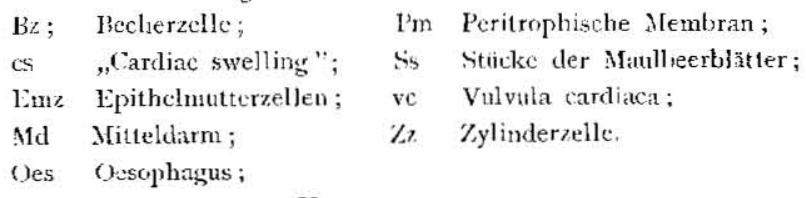

Vergr. 70: I 
Neuerdings ist ein Beweis von SiIto (1930) erbracht worden, dass dic Zellen des Mitteldarmepithels dimorph sind, obwohl or cigentlich die Homomorphie-Ansicht von SHINODA teilt. Er beobachtete ungefähr 20 epitheliale Anschwellungen ("Cardiac swelling" genannt) im Sphinkterabschnitt bej Antheraea pornyi (Lepidoptere). Fir hat festgestellt, dass dieser Teil des Mitteldarmepithels nur aus den sezernierenden Zylinderzellen besteht. Weiter hat er auch einige solche epitheliale Anschwellungen bei Bomby $x$ mori gesehen. Sie sind jedoch nicht so deutlich erkennbar wie bei Anth. pernyi. In den Längsschnitten durch den Darm von Bombyx mori fand ich, dass etwa zehn erste Zylinderzellen am vorderen Ende des Mitteldarms höher sind (Abb. 3). Vielleicht entsprechen sie dem „Cardiac swelling." Hier bcobachten wir nie Bccherzellen, wenn sie auch ihren Inhalt ins Darmlumen sezerniert haben.

Darum glaube ich, dass die Zellen des Mitteldamepithels dimorph sind.

ÜBER DIE ENTSTEHUNG DER EPTTHELZELYEN DES MTTELDARMS

IM EMBRVO

VERSON (1905) untersuchte die Fntstehung der Epithelzellen des Mitteldarms bei Bombyx mori. Er fand aber in diesen keine Becherzellen, sondern nur Zylinderzellen und Nidi. Dagegen hat Tchang Yung-TaI (1928a, I929b) im Fmbryo von Galleria mellonella sowoil Zylinderzellen wic Becherzellen beobachtet. Nun wollen wir die Iintstehungsweise der Epithelzellen des Mitteldarms bei Bombyx mori nochmal eingehend und genau verfolgen. Die Entstehung des Mitteldamejithels geht von den entodermalen Zellen aus einige Tage vor den Ausschlïpfen der Raupen. An Vormittag 3 Tage vor dem Ausschlüpfen zcigt der Fmbryo noch keine Pigmente am Körper. Die Öfnung des Mitteldarms ist für den Dotter noch niclit zugeschlossen, wodurch noch ein Menge von Dottergranula und Dottcrzellen ins Lumen des Mitteldarms hineindringt. In clem vorderen und hinteren Ende des Darmrohrs schliessen sich das Stomodacum und das Proctodacum, welche aus den ectodcrmalen Zellen schon früher als der Mitteldarm gebildet wurden. Stonodaeum und Proctodaeum nehmen die Gestalt von Flaschen an, die ihren Boten nach dem Mitteldam richten. Der Boden ist durch ein sehr dünnes einschichtiges Epithel gebildet und sperrt den Verkehr zwischen dem Mittel- und Vorderoder Hinterdarm ab. Dabei besteht die epitheliale Wand des Mittel- 
darms aus unregelmässigen, auseinanderdrängten und noch hon:omorphen rundlichen Zellen (Tafcl 2. Abb. 13). Diese Zellen baben verhältnissmäsig grosse Kerne in Zentrum. Ihr Plasma ist homogen. An vielen Stcllen findet man mitotische Figuren, deren Kerntcilungsspiudeln keine bestimmte Richtung haben. In Stadium der Metaphase war eine Zahlung der Chromosomen unmöglich. Allerdings scheinen die Mitteldamepithelzellen in diesem Stadium sich im ganz embryonalen Zustand zu befinclen. Sic weisen noch keine morphologisclıe Differenzierung der Zellarten auf wic in der Larve, nämlich in Zylinder\%ellen und Becherzellen. Am Nachnittag dieses Tages bemerkt man schon schwarze Pigmente in ten Ocelli und der Mandibel des Embryo, und die Finmündung des Darmrohrs wird geschlossen. Es scheint, dass die Dottergranula sich im Lumen aufzulösen beginnen, besonders in der Umgebung der Dotterkerne. Wesentliche Veränderungen sind mit den Epithelzellen des Mitteldams sich gegangen. An der Epithelwand bemerkt man jetzt regelmässige, nebencinander liegende, cine einfache Schicht bildende Zellen und es kommen die zweierlei Zellformen zum Vorschein (Tafel 2, Abb. 14). Dic cinen sind kubische: und höhere Zellen und zeigen ovale, in der Mitte der Zellen liegende Kcrnc. Ihr Plasma ist nicht so färbbar. In distalen Teil, mit dem das Dotter in Berührung kommt, zeigen sic sich ein bisschen acidophil. Nun schcinen sie den Dotter lebhaft $\%$ absorbieren. Die anderen Zellen stellen sich aber keilförmig dar; sie sind hin und her zwische. den Zylinclerzellen gelagert. Ihr Plasma wird mit dem Hämatox' in stark grefarbt, wodurch wir sie von den '/ylinderzellen sehr leicht 1 nterscheiden können. In diesem Stadium ist weder der Stäbch nsaum noch Sekretion sichtbar. An älteren Stadien, nämlich 2 lage vor dem Schlüpfen, schrcitet die Differenzierung der zwei deutlich anterscheidbaren Zelltypen weiter fort. Nun können wir sicher er snmen, dass die keilformigen Zellen die Becherzellen sind. Die Spit" - dieser Zellen wird allmäblich inmer dicker, und an diescr Stellr trittc ein mit SäureFarbstoffen stark färbbarer Teil auf. In die,em Stadium verschwindet dic dünne Membran, die den Boden der Köhren von Stomodaeum und Proctodacum bilkete, wodurch der Jamiang zu einer langen offenen Röhre wird. Gleichzeitig wird , 1er Dotter, welcher ausscrhalb des Raupenkörpers übriggebliebr.. ist, und die Scrosa durch den Mund in den Darm so reichlir-1, cingeführt, dass dic Epithelzellen sich wegen des Nahrungszuf asses spannen und etwas niedriger werden.

Ein $1 \%$ vor dem Schlüpfen wird der Embryokörper infolge viełer 
Pigmente sehr dunkel. Der Inhalt des Darms wird sehr spärlich. Nun beginnt der Stäbchensaum über den Zylinderzellen zu wachsen (Tafel 2, Abb. 15). Das Plasma der Zylinderzellen nimmt an granulären Bestandteilen zu. Häıfig sind einige Vakuolen darin enthalten. Die Safthöhlung der Becherzellen vergrössert ihr Volumen nach unten zu. Infolgedessen wandert der Kern nach unten und wird in hohem Gracle flachgedrückt. Wir können die hin und her verlaufenden Epithelmutterzellen in der basalen Membran sehen.

Vor dem Schlüpfen der Larve hat Verson (1905) bei Bombyx mori und Tciang Yung-Tai (1929c) bei Galleria mellonella die Degeneration und die Eneuerung des Mitteldarmepithels beobachtet. Diese Tatsache konnte ich nicht feststellen, trotzdem viele Fmbryonen untersucht worden sind.

I'rüh am Morgen, kurz vor dem Schlüpfen der Larve aus dem Ei, kommt das Abstossen vieler Sekretblasen ins Darmlumen zum Vorschein. Die Blase ist basophil und voll von Granula innerhalb des Häutchhens. Wir haben jetzt typische sezernierende Zylinderzellen und die Becherzellen vor uns. Somit haben sich die zweierlei Arten von Zelltypen des Mitteldarmepithels beim kmbryo unabhängig von einander entwickelt.

Wir können also auch auf Grund dieser Tatsachen sagen, dass die Zellen des Mitteldarms dimorph sind.

\section{CEBER DIE ENTSTEHUNG DES MITTEI.DARMEPITHELS WḦHREND \\ DER HÄUTUNG}

Vorbereitungsstadium I (20-34 Stunden vor der Häutung).

In diesem Stadium ist das Darmlumen mit dem Futter (Maulbeerbaum) noch ganz gefüllt. Ueber die Zylinder- und Becherzcllen ist im allgemeinen nichts neues zu sagen, nur dass die Sekretion nicht so lebhaft ist wie im normalen Zustancle. In der oberen Zellhälfte treten jedoch mehr oder weniger unregelmässige Körnchen auf. Stäbchensaum und basale Körnerreihe sind überall gut erhalten. Wesentliche Veränderungen finden mit den Epithelmutterzellen statt. Im Anfang jeder Raupenperiode isolieren sich die Epithelmutterzellen als Inselgruppen oder Zweige, dann vermehren sich die Zellen durch rege Teilung wahrend der betreffenden Periode. Gegen den Anfang der Häutung (während des Vorbereitungstadiums) verbinden sich die Inseln oder 
Zweige miteinander und bilden cin Netzwerk. Alb. 4-7 zeigen tangentiale Schnitte aus dem Darmgang. Abb. 5 stamnt von Raupen 34 Stunden vor der Häutung. Wic sie zeigt, sind dic Gruppen der Epithelmutterzellen noch nicht vollkommen miteinander verbunden. Abb. 7 gehört aber $z u$ einem weiteren Stadium, nämlich 28 Stunden vor der Häutung. Hier sind die Epithelmutterzellen-Gruppen ganz dicht verbunden und bilden ein vollkommenes Netzwerk. In Längsschnitt sieht man die Zellen in den Gruppen zusammen; sie sitzen mit der breiten Basis der Lasalmembran an. Ihre scharfen Spitzen dringen in dic alten Epithelzellen bis zu etwa $1 / 3$ ihrer Ilöhe hinein. Ihre Kerne sind kreisrund; sie betragen etwa $1 / 4$ des Durchmessers der Zylinderzellenkerne, sind also bcdeutend kleiner. Ihre Lage ist abcr sehr verschieden, zum Teil zeigen sie einen Hof, zum Teil keinen. Häufig findet man hier Kernteilungsfiguren.

Vorbereitungsstadium II (20 Stunden vor bis zur 1 Iäutung).

Wenn die Raupe mit dem Beginn der Abscheidung ciner neuen Cuticula in den eigentünlichen Ruhczustand eintritt, frisst sie nicht mehr, und der vordere Teil des Mitteldarms ist daher fast leer. Die peritrophische Membran ist einschichtig, aber selrr dick. Einc selur lebhafte Sekretkugelabscheidung wircl nur im hinteren Teil des Mitteldarms beobachtet, und da zeigen die Sekretblassen reichliches Vorhandensein feinster Körnchen. Im allgemeinen befinclen die Zylincier-

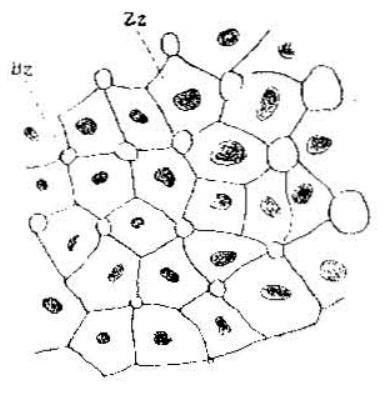

Aib. 4 .

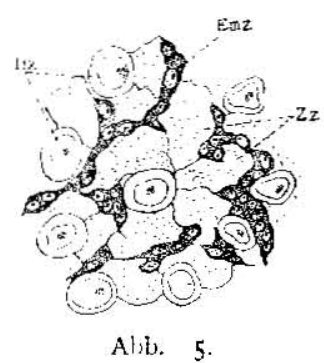

Abl. 4 und 5. Zwei tangentiale Sclinitte aus dem Iharmgang 34 Stunden vor der ersten ILïutung. Sie zeigen die Verteilung der Zylinder. und litechersellen.

Abb. 4 stellt einen Quersclinilt durch die Zy!inderseller nit ihren Kernen und durch die oberen Teile der Becherse!len dar.

Abb. 5 stelit einen Sclinit dicht an der Basalmenibran dar (aus demsellon Epithel). Vergr. $980: 1$

Bezcichnung wic in Abb. 1 . 


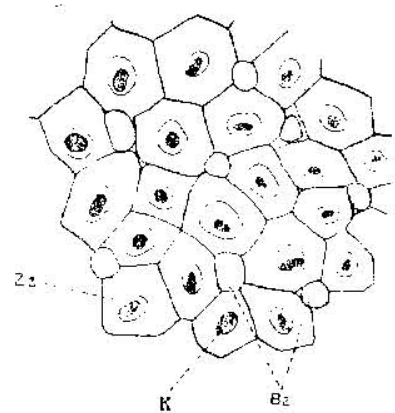

Abb. 6 .

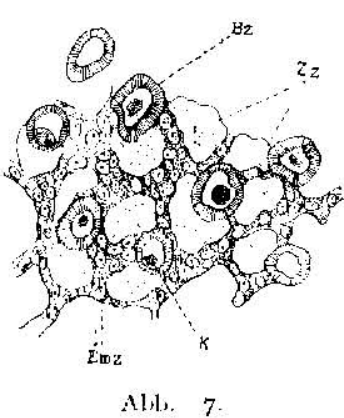

All. 7 .

Abb. 6. Wì Alb. 4, 28 Stunden vor der ersten IIäutung.

Ably. 7. Wie Alt3. 6, dex Schnatt ist aler durch den basalen Mcurbran diche anliegenden Teil gefülurt.

Vergr. 2030:1

Bezeichnungen wie in Abb. $x$.

zellen sich in Zustand der Kuhc. In der Safthöhlung der Becherzellen treten die unregelınässigen, schwarz gefärbten Fiuschlüsse häufig auf. Die Kerne beiter Zellarten weisen nach Lage und Bau keine Veränderung anf. Jie Epithelmutterzellen haben jetzt nahezu $1 / 22 / 3$ Höhe des Epithels erreicht. Gegen das Ende dicses Stadiums wird eine Differenzierung in Zylinderzellen und Becherzellen in der Gruppe der jugendlichen Iipithelmutterzellen sichtbar. Dabei benerkt man im zugespitzten Teil der jugendlichen Zcllen, dic sich zu Becherzellen entwickeln, einen acidophil färbbaren Teil (Tafel 2, Abb. I6).

Häutungsstadium bis unmittelbar nach der Häutung.

Der Mitteldarm ist ganz leer. Die peritrophische Membran wird inmer dicker und mehrschichtig. Sekretblasen sind nur noch hier und da in vorderen und im hinteren Abschnitt des Mittcldarms bcobachtet worden. In diesem Stadium nimmt lic Grösse der Zylinelerzellen infolge plötzlichen Wachstums $z u$, wodurch ihre Zellfront stark nach aussen ausgebuchtet wird (Tabel 2, Abb. 17). Daneben vollzielst sich ein starkes Wachstum des Darmgangs durch das I linzufügen jugendlicher Zellen. Dic Bcchcrzellen zcigen noch kein abweichendes Verhalten von demjenigen des letzten Stadiums. Die beitlerlei jugendlichen Zelien haben fast alle die IHöbe des Epithels erreicht und ilre Differenzierung geht weiter vor sich. In der jugendlichen Becherzelle kommt es mun zur Ausbildung einer so dentlichen Safthöhlung, dass wir jetzt die typische Becherzelle vor uns haben (Tafel 2, Abb. 17). Es ist 
noch sehr leicht, die jugendlichen Zellen von den alten zu unterscheiden, demn dic jugendlichen Zellen sind noch kleiner als die alten (Abb. 8).

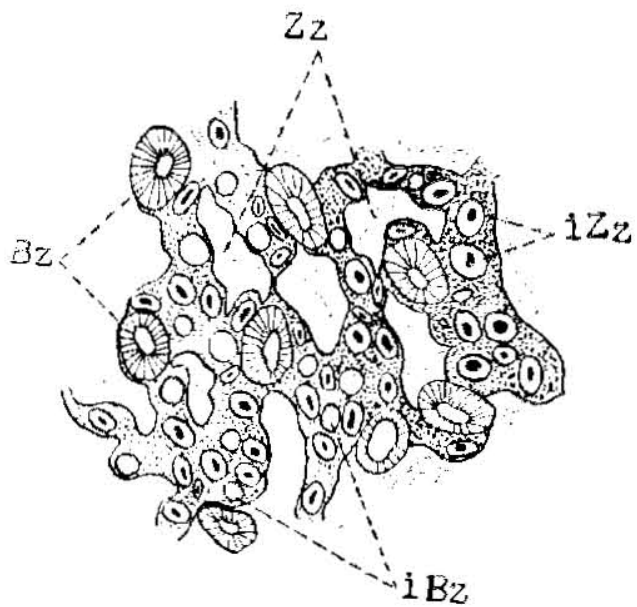

Abb. \& Wie Abb. 5, 7; 4 Stunden nach der ersten Itäulung. Hier kann man die differenzierten jungen Zylinder- und Becherzellen beolrachten.

\begin{tabular}{ll}
\multicolumn{3}{c}{ Vergr. 650: 1} \\
B. Recherselle; & iZz junge Zylinderzellon : \\
ilz junge Becherzellen; & 7.2 Zylinderzellen.
\end{tabular}

Während der läutung werden die aus dem Epithel ausgestossenen Zclien in grösserer Zahl als in normalen /ustand beobachtet. Ich habe dabei eine totale Epithelausstossung nicht feststellen können, die Verson (1897) und Tchang Yung Tal (1928, 1929), jener an Bombyx mori, dieser an Achroca grisella und Galleria mellonella konstatiert haben. Fs scheint mir auch bei Bombyx mori nicht schr wahrscheinlich zu sein, dass die Fpithelerneuerung kontinuierlich ist, wie SHrvond (1926) und BuCHMANx (1928) angeben.

Es entsteht also die neue Epithelwand des Mitteldarms in jeclem Wachstumsstadium aus den periodisch hinzugefügten jugendlichen Zellgruppen, die sich aus Epithelmutterzellen entwickeln, wic ILxsos (1929) festgestellt hat, zwischen den alten Fpithelzellen Dabai ist es bemerkenswert, dass die Zylinderzcilen und die Becherzellen unabhängig voneinander aus den Epithelmutterzellen gebildet werden.

Es grenügt dieser Hinweis, meiner Ansicht nach, um dic Vermut- 
ung auszusprechen, dass das Mitteldarmepithel bei der Seidenraupe und damit überhaupt bei den Lepidopteren-Larven cin dimorphes ist.

DAS MITTELDARMEPI'HEL DER SEIJENRALFEN WRHREND

DES HCNGERNS

Nach 48-stündigem Hunger hat der Mitteldarm nur ein wenig Speisc im Lumen, dic von einer dicken mehrschichtigen peritrophischen Membran umgehüllt worden ist.

Dic Sekretion findet selir lebhaft statt, besonders in vorderen und im hinteren Teil des Mitteldatms. Diese Erschcinung stimmt mit der Beschreibung von JokDan (1912) überein. 1)ie Sekretblasenbildung der Zylinderzellen zeigt keine Abweichung vom normalen Verhalten. Is ist aber sehr merkwürdig, dass der acidophile Saft in der Höhlung der Becherzelle nun zu verschwindon beginnt, trotzden wir den Austritt eines sekretähnlichen Saftes aus dieser Höhlung ins Darmlumen hinein nicht beobachten konnten. Was dic physiologische Bedeutung der Becherzelle anbelangt, sind noch weitere eingehende Untersuchungen erforderlich.

Nach 72-stündigem Hunger beginnt das Plasma in der Zylinderzelle in ihrer unteren Hälfte etwas acidophil $z u$ werclen und ist bei der Becherzelle um so viel spärlicher geworden, dann der Kern zwischen der Safthöhlung und der Basalmembran eng eingeklemmt crscheint.

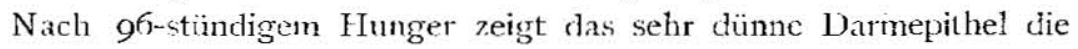
mageren Zylinderzellen. Der Kern der Zylinderzellen farbt sich mit Hämatoxylin gan\% schwar\%, weist aber in Bez. auf dic I.age keinc Veränclerung auf (Abb. 9).

Ihr Plasma ist jetzt fast acidophil, und die im Ylasma verlaufenden granulären Stränge sind unregelmässig. Dic Becherzellen sincl gross wie sonst; thr Safthohlraum hat scincn Inhalt ganz entleert. Der Stäbchensaum ist überall deformicrt. Dic Sekretabstossung kann man nur in hinteren Teil des Darmepithels beobachten.

Dic Epithelnutterzcllen sind empfindlicher als andere Elemente für die Iungrerbeschädigung; sie unterbreclen schon naclı 48-stünligem IIunger ihr Wachstum und ihre Kerne ziehen sich zu ciner schwarz gefäbten degenerierenden Chromatinmasse zusammen. Nach 144stündigem Hunger sind alle Epithel\%ellen ganz acidophil mit Ausnahme der Kerne, und die degrenerierten uncl abgestossenen Zellen fliessen überall in das Iumen hinein. 


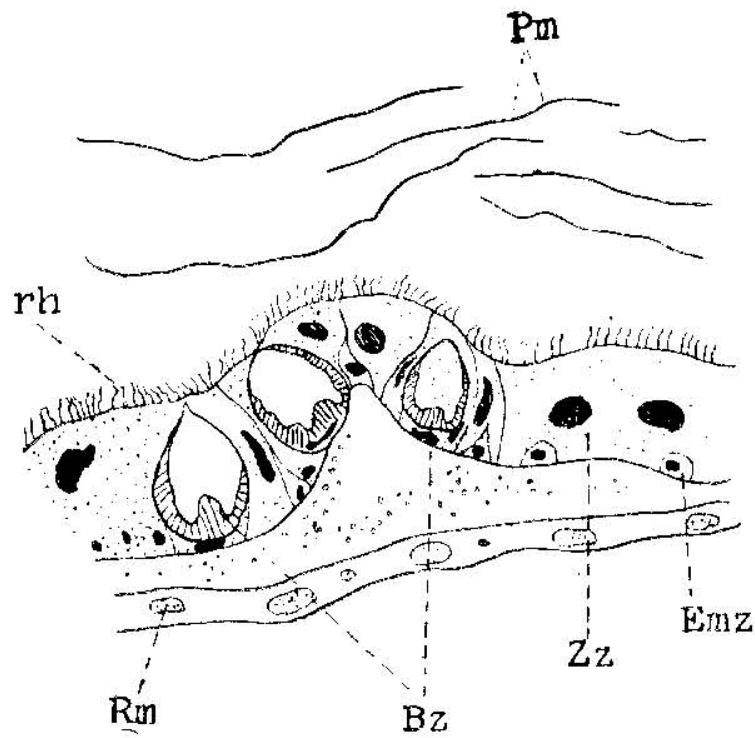

Abb. 9. Epithelzellen nach 96 stiindigem Hunger. 3 Tage alte Larse in dritten Wachstumsstidium.

Vergr. $560: 1$

Bezeichnunges wie in $\mathrm{Abb}$, I und 3 .

Bei dem Degenerieren der Epithelzellen während des Hungers konnten wir nie Uebcrgänge von Zylinderzellen zti Becherzellen beobachten.

\section{VERTEILUNG I)ER BECHERZLLLLN UNI) DER ZYLINIERZELLEN}

MI MTIELARMEITHEL

Bei den tangentialen Schnitten durch den Mitteldarm sehen wir die Verteilung der Zylinder und der Becherzellen im Dormepithel. Im Embryo verteilen sie sich vor dem Schlüpfen so, dass eine Becherzclle von etwa $4^{-6}$ Zylinderzellen umgeben, ist wie Abb. 10, i r zeigen

In derselben Weise gestaltet sich dic Vertcilung der Epithelzellen nicht nur in normalen Zustand der Wachstumsstadien der Ralupen sondern auch während und kurz vor oder nach der IIäutung, oder auch nach langem Hunger (Abb, 4-8, I2).

Aus dem Vorhergehenden könnnen wir schliessen, dass die Verteilung der Zylinder- und der Becherzellen kcine Beziehung zur Sekretionserscheinung hat. Es erweist sich also, dass dic Zellen des Mittelclarmepithels bei der Seidentaupe dimorph sind. 

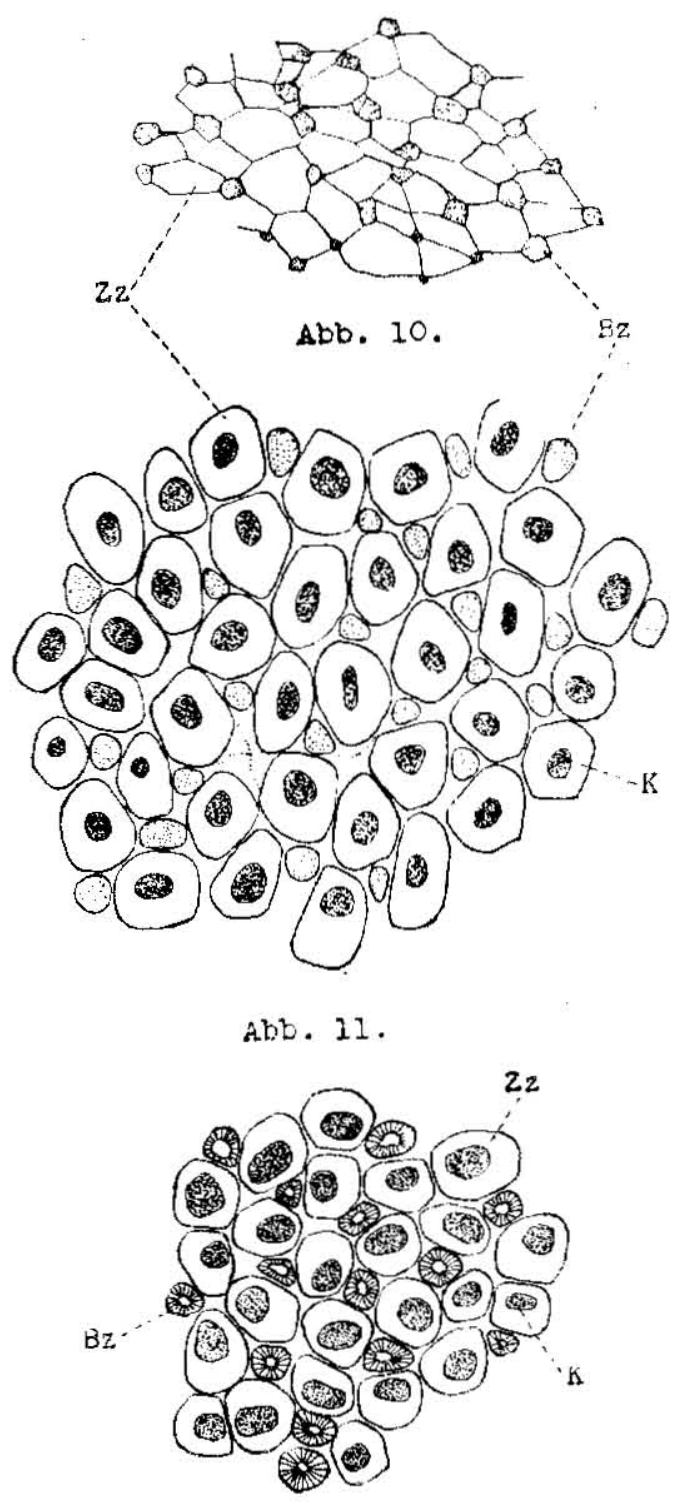

Att. 1?.

Abb. 10. Verteilung der Zylinder - und der Bechergelten in Mitteldarmepithel des Enbryos 3 Tage vor dem Ausschliupfen.

Alb. I1. Wie Abl. ro, 2 Tage vor dem Schliipfen.

Abb. 12. Wic Abb. Io u. II. Aus einer eben herausgeschlitpften Raupe. V'ergr. 956: I

Bezeichnungen wie in Abb. 1 . 


\section{ZUSAMMENTASSUNG}

1) Es ist anzunelmmen, dass die Sekretion des Mitteldarmepithels bei der Seidenraupe cine meracrine ist, wie sie von Sunnona (1926) bei cinigen Lepidopteren festgestelit wurde.

2) Die Epithelemeuerung und das Epithelwachstum beruhen auf periodischen Hinzufügen von jugendlichen Zellen, die sich aus den Epithelmutterzellen entwickeln, zwischen den schon vorhandenen Epithelzellen, und danebon auf dem Wachstum jeder Zelle während der Raupenstadien. Dabei habe ich eine totale Epithclausstossung nicht feststellen können, die Vkrson (1898) und Tchang Yung-Tar (1929, 1629) konstatiert haben.

3) Was die Frage der Morphogenie der Epithelzellen anbelangt, glaube ich auf Grund folgender Tatsachen, dass die Epithelzellen bei der Seidenraupe dimorph sind :

a) Im normalen Zustand bin ich keinen Figuren von Uebergängen von Zylinder- zu Becherzellen begegnet, welche von Vrason (I905) für bombyx mori, von Sinnona (1929) für Caligura japonica und einige Lepidopteren-Raupen und von Btchman (1928) für die PyranstaRaupen angegeben werden.

b) An vorderen Ende wird das Mitteldamepithel nur aus hohen Zylinderzellen gebildet und hier haben wir nie Becherzellen in Sekretionstätigkeit oder Ruhezustand beobachten können.

c) 3 Tage vor dem Schlüpfen des Jimbryos aus dem Ji.i finden wir schon dic Differenzierung seiner Mitteldarmepithelzellen und zwar in Zylinderzellen und in Becherzellen, trotzdem sie noch keine Sekretionstätigkeit ausüben.

d) Während der jläutung konnten wir beobachten, dass die Zylinderzellen und dic Becherzellen unabhägig voneinander, jeder Typus für sich, aus den Jipithelmutterzellen entstehen.

e) Bei dem Degenerieren der Zylinderzellen nach langen Hunger gehen diese nicht in Becherzellen über.

f) Die Verteilung der Zylinder- und der Becherzellen in Mitteldarmepithel bleibt fast unverandert sowohl beim fimbryo wie in allen Wachstumsstadien der Raupen und auch im Hungerzustand.

\section{LITERATUR}

I. Bralin, M. (19rz), Das Mitteldarmepithel der Inscktenlarven wälırend der ITäutung. Zeitschr. f. wiss. Zool. Jid. 103, Ht. I. 
2. TitchmaxN, W. W. (1928), Zur Ernährungsphysiolngie normaler und hungernder Pyyarrsta-Raupen. Zirol. Anz., Bd. 7, IIt. 7/8.

3. Dicklikik, I'. (1900), Beiträge zur Kenntnis der Damsekretion. I, Deillophiza cuthorline. L. Arch. f. Naturg., Jahrg. 75.

4. - (1913), Der Iarmtraktus und seine Anhänge. Handlouch der Entomolngie, Crkt. SCHRöntr, Jena.

5. FoA, A. (Igr8), T'epithelin dell' Intestim medio nel bacn da seta samo e in quello malato di flaccidezza. Rend. d. Institutn Bac. d. R. Sc. sujer. di Agric, in Portici. Vol. 3

6. Jikniki, J. (1885), Finiges über den Mitteldarm der Insekten snwic über Kpithelregeneration. Arch. mikr. Anat., Bd, 26.

7. IAstaman, 1.. (1910), The structure and metamorphinsis of the alimentary canal of the larva of I'sychedit alternati. Ann. Entom. Soc. Amer. 3.

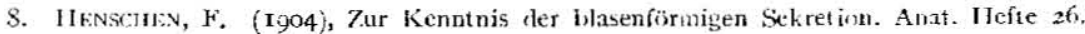

9. IIt:Nsos, IT. (1929), On the development of the mid.gut in the larval stage of Inmessa whirae. Q.J.M1.S. Vol. 73, Nis. 289 .

Io. JoRnAN, If. (1913), Verglcichende Physiolngie wirlselloser Tiere. I Bd. Frnährung.

11. - und Stratink, A. (1911), Ueber dic sekretive und alsortive Funktion der J)armzellen hei Wirhellasen inshesndere bei Insekten. Verhandh, deutsch. Zaxisl. Ges.

12. Nincompr, l. J. (1914), Sime notes on the digestion and the cell structure of the digestive epritlelium in insects. Ann. Entom. Sioc. Aner. 7.

13. Suro, S. (rojo), A report on the cardiac swelling of the midintestine of the Tusserworm (Antheroed fornyi Gurk.). Transact. Siapioro Nat. IIist. Soc., Vol. is, Ht. a.

14. SHIsoda, (). (1926), Contributions is the ktowledge of the intestinal secretinn of instets. J. Mid-intestinal secretion of Lepidiptera with an appendix; Relavior of mitnclusulria in the mid iritestinal epillelium of the silk-worm, Fontix mori, $\mathrm{L}$. Mem. Coll. Sci. Kyot Imp. Univ, St:r, B, Vol. 2, No. 2.

15. - - (1927), Contributions to the knowledge of intestinal secretion in insects. II. A comparative listo-cytolugsy of the mid-intestine in varinus orders of insects. Zellf. u. mikr. Anat., Bd. 5 , Ht. 3 .

16. Toming: Vung-TAi (1928 a), Sur l'origine, le développment et la structure des cellules calicifirmes dans l'intestin minyen des chenilles de Lépidoplères. Bull. Soc. Zixol. de France, t. 103.

17. - - (1928 b), Les rémovations successives (partielles ou tonales) de l'épithélium do l'intestin myen chez les clienilles de Calleria mellonelh. C. R. Sixc. Biol., 1. 93.

38. - - (1928 c) La structure du tube digest if et les rénovations successives de l'épitlélium de l'intestin myen chez les chenilles d'Achroia (nu Achroeal) grisella J. Ibid.

19. — (1929 a), La structure et les rénovations successives de l'épithélium du mésentéronn chez lembryon du liepidnptere: Girlierio melionella. C. R. Snc. Biol, t. roo

20. - (1929 b), Recherches sur l'histogenése et l'histophysingngrie de l'intestin monyen chez un Lépidoptère (Gratieria mellonella L.). Suppl. Bull. Bins. de France et de Jiejgique $\mathbf{2}$.

21. VAN (iEHUCh'ten (1890), Recherches histolngiques sur l'appareil digestif de la $7^{2} y^{\prime}$. choptera contoninata. Cellule, t. 6.

22. VERSoN, E. (1897-I898), Zur Entwicklung des Verdaungskanals beim Seidenspinner. I. it II. Zoni. Anz., Bd. 20-2r.

23. - (1905), Zur Entwicklung des Darmkanals Bombyx mori. Zeitschr. f. wiss. Zool., I3d. 82 . 


\section{TAFELERKLÄRUNG}

Alle Abbildungen auf Tafel I sind nach Schnittpräparaten von in CARnors Chloroform-Fisessig-Alkohol fixiertem und mit Hämatoxylin nach Driskieco und Eosin doppelgefärbtem Darmepithel angefertigt.

A7h. 13-15 wurden mit ZFiss Oel Inmers. I/12 und ZFiss Kompens. nkular 8 und Abb, I6-17 mit Objectträger D und ZfIss Kmms, nkular 8 unter Benützung des Abbeschen Zeichenapparates trei Onjekttischhïhe gezejchnet. Tei der Reproduktion wurken sie $3 / 4$ verkleinert, sodass sich cine Vergrössung von etwa $75^{\circ}$ in Alh, 13-15 und 300 in Abb. 16.17 ergsilit.

Abl. I3-15. Iängsschnitt durch den Mitteldarm von drei Embryonen itm ansteigenden Alter,

Abb. r3. Aus einem: Fmbryo in Morgenfrühe drei Tage wor dem Ausschlüpfen.

Abl. 14. Nus einem Fmbryo nachimittag drei Tage vor dem Ausschliupfen.

AŁb. 15. Aus einem Embryo einen Tag vor dem Ausschlüpfen.

Abł. 16-17. Mitteldarmepithel während der IJäutung.

Albb. 16. Drei Stunclen vor der ersten Fäutung.

Abb. 17. Zwei Stunden nach der ersten Vläutung.

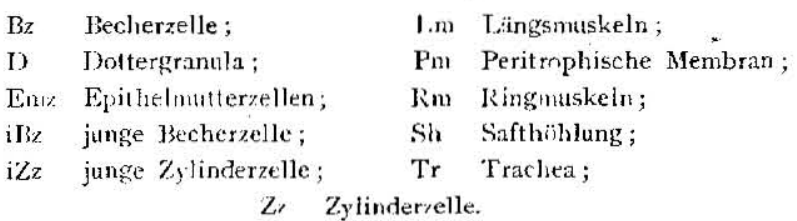

Z, Zyinder,elle. 
Abb. 15 .
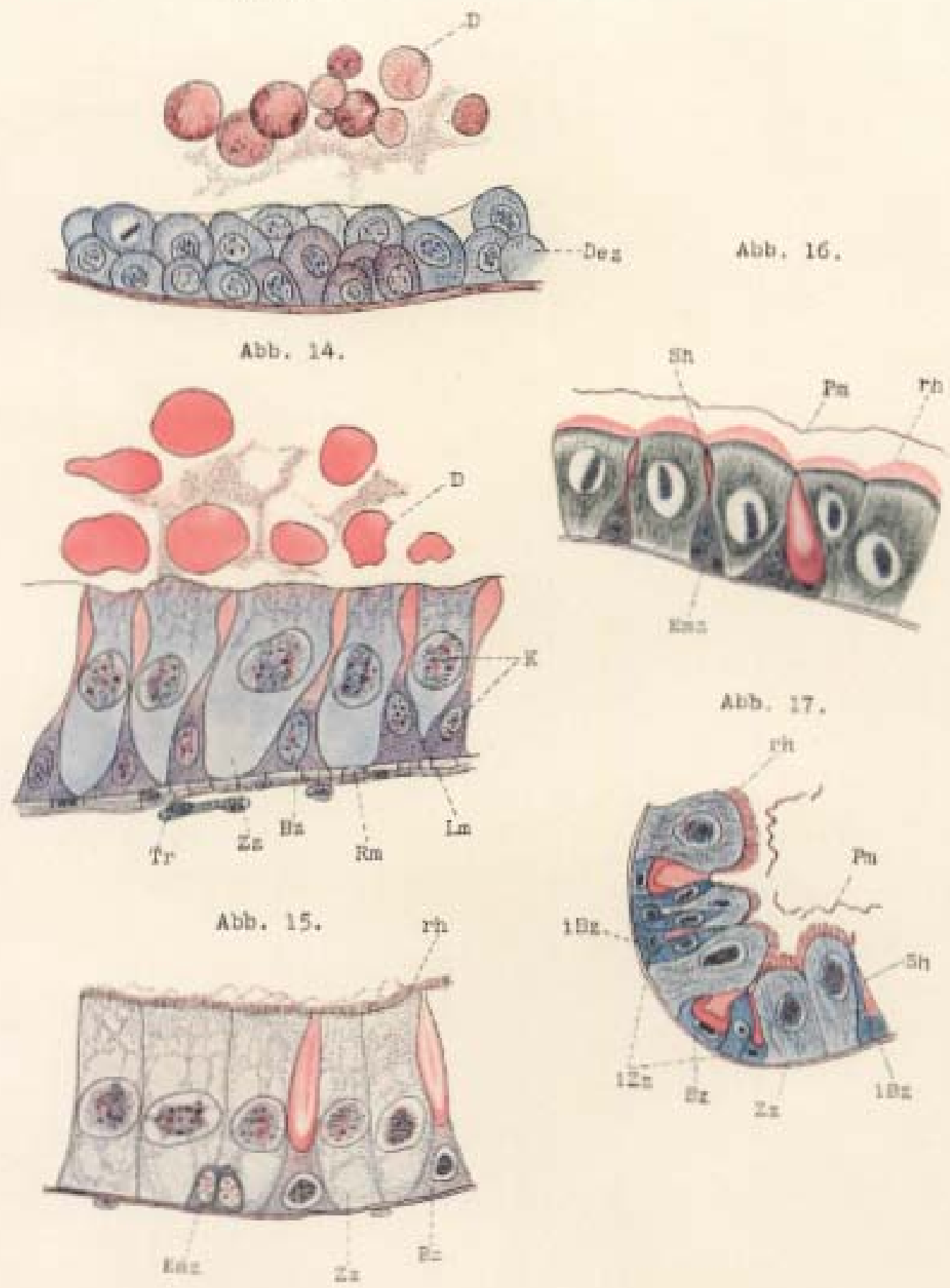